\title{
Sexuelle Zufriedenheit von Frauen - Entwicklung und Ergebnisse eines Fragebogens
}

\begin{abstract}
Zusammenfassung: In der Sexualforschung erfassen die vorhandenen Fragebogen die Erlebnisebene der Sexualität und den Grad der sexuellen Zufriedenheit nicht hinreichend differenziert. In einem eigenen Projekt wurde daher ein Fragebogen entwickelt, der als Basisdaten die Häufigkeit und Dauer der sexuellen Handlungen, ferner die Zufriedenheit mit Häufigkeit und Dauer dieser Handlungen sowie das erwünschte Sexualverhalten berücksichtigt. Eine erste Untersuchung mit dem Fragebogen an 112 Frauen mit heterosexuellem Sexualverhalten im Alter von 20-48 Jahren wurde durchgeführt. Die hohe Wunschrate bezüglich des koitalen Orgasmus unterstreicht die Wichtigkeit des Orgasmuserlebens. Andererseits nennt die Hälfte der befragten Frauen den Orgasmus nicht als ihre favorisierte Empfindung beim Geschlechtsverkehr. 37\% der Frauen geben explizit an, dass die emotionale und körperliche Nähe zu ihrem Partner wichtiger als das Erleben des Orgasmus ist. Gemäß dem Extremgruppenvergleich korreliert sexuelle Zufriedenheit vor allem mit der in der Partnerschaft realisierten Selbstbestimmtheit, der Befriedigung der Kommunikationswünsche innerhalb der Partnerschaft und des Zärtlichkeitsbedürfnisses.
\end{abstract}

\begin{abstract}
Sexual Satisfaction of Women - Development and Results of a Questionnaire: In sexology the existing questionnaires do not sufficiently consider the experiencing of sexuality and the extent of sexual satisfaction. That is why a questionnaire was developed which includes, besides the frequency and duration of sexual activities, the satisfaction with frequency and duration of these activities and the desired sexual behaviour. A first study with this questionnaire was carried out on 112 women with heterosexual behaviour, aged 20 to 48 years. The frequent desire with regard to coital orgasm as one result of our investigation confirms the centering of orgasm in other studies. But half of the women do not describe orgasm as the favoured feeling during sexual intercourse. For $37 \%$ of the women the emotional and physical closeness to the partner is explicitly more important than experiencing an orgasm. According to the comparison of extreme groups sexual satisfaction particularly correlates with self-determination realized in partnership and with satisfaction of communicational desires and need for tenderness within the partnership.
\end{abstract}

PPmP Psychother Psychosom med Psychol 2001; 51: 68-75 (c) Georg Thieme Verlag Stuttgart · New York

ISSN 0937-2032
Sabine Büsing, Claudia Hoppe, Reinhard Liedtke

Klinik und Poliklinik für Psychosomatische Medizin und Psychotherapie der Universität Bonn (Direktor: Prof. Dr. med. Reinhard Liedtke)

Key words: Sexual satisfaction of women - Questionnaire Comparison of extreme groups

\section{Einleitung}

Die vorliegende Arbeit entstand im Rahmen eines Forschungsprojektes zur „sexuellen Zufriedenheit von Frauen und Männern" der sexualmedizinischen Ambulanz der Universitätsklinik für Psychosomatische Medizin und Psychotherapie Bonn. Hauptgegenstand dieses Projektes ist die inhaltliche Erfassung des Begriffes „sexuelle Zufriedenheit“, ihrer Kennzeichnung und ihrer Bedeutung. Von primärem Interesse sind dabei nicht die quantitativen Aspekte des Sexualverhaltens im Sinne von Häufigkeit und Variationen, sondern das Erleben der Sexualität und die daraus resultierende Zufriedenheit.

Zur Erfassung der sexuellen Zufriedenheit existiert in der Sexualforschung eine Reihe von Fragebogen, in denen jedoch die Erlebnisebene der Sexualität und der Grad der sexuellen Zufriedenheit nicht hinreichend differenziert erfasst wird [1]. Meist findet die sexuelle Zufriedenheit nur auf Subskalen Beachtung, wie beispielsweise in der deutschen Übersetzung des Marital Satisfaction Inventory von Schröder et al. [2]. Die Mehrzahl der Instrumente zielt darauf ab, Aussagen über das durchschnittliche Sexualverhalten oder das Bestehen sexueller Schwierigkeiten zu ermöglichen [3].

Thema dieser Arbeit ist die Konzeptualisierung, Erstellung und Erprobung eines Fragebogens zur Erfassung der sexuellen $\mathrm{Zu}-$ friedenheit von Frauen. Der Fragebogen soll nicht in der Tradition der Sexualreporte Daten über das Sexualverhalten liefern. Dennoch soll der quantitative Aspekt des Sexualverhaltens erfasst werden. Die Häufigkeitsdaten sexueller Aktivitäten dienen als Weg, sich den Zufriedenheitsdaten zu nähern, sie zu interpretieren und relativieren zu können.

Für die sexuelle Zufriedenheit als ein überdauerndes Gefühl muss eine Abgrenzung von der aktuellen sexuellen Befriedigung erfolgen. Empirische Arbeiten zur sexuellen Befriedigung

Eingegangen: 5.5.99

Angenommen: 1.3 .2000 
erfassen diese als kurzfristigen emotionalen Impuls, der auf situationsspezifischen Bedürfnisbefriedigungen beruht. Sexuelle Zufriedenheit als überdauerndes Gefühl dagegen erwächst nach Levine [4] aus der Balance der partnerschaftlichen Sexualität, aus der Fähigkeit, das Sexualleben mit nicht sexuellen Aspekten der Beziehung zu integrieren sowie aus den persönlichen Erwartungen an das Sexualleben. Dies bedeutet, dass sexuelle Zufriedenheit trotz sexueller Dysfunktionen entstehen kann, das Fehlen solcher Dysfunktionen jedoch kein Garant für sexuelle Zufriedenheit sein muss.

Die bekannten Sexualreporte der Vergangenheit mit Angaben zu Häufigkeiten und Vorlieben bezüglich des Sexualverhaltens lassen größtenteils die Fragen nach dem Erleben von Sexualität und speziell von sexueller Zufriedenheit offen. Unter den Arbeiten zur weiblichen Sexualität ist der Hite-Report hervorzuheben. Shere Hite $[5,6]$ legte eine differenzierte Studie vor, die sich mit weiblichem sexuellen Erleben beschäftigt, ohne männliches Erleben definitorisch und normativ zugrunde $\mathrm{zu}$ legen.

Die von von Sydow [7] befragten Frauen berichteten von zu wenig Zärtlichkeit und zu häufigen Koituswünschen ihrer Partner. Der seltenere Wunsch der Frau nach Koitus [8] lässt jedoch nur dann auf eine geringere weibliche Libido schließen, wenn Libido als Lust nach Verkehr und Orgasmus definiert wird. Betrachtet man die Häufigkeiten der Wünsche nach Zärtlichkeit, zeigen die Frauen in allen Altersgruppen ein stärkeres Verlangen als die Männer. So schätzen Frauen auch die partnerschaftliche Übereinstimmung sexueller Wünsche als geringer ein als Männer. Männer bestimmen heute noch meist das „Wann und Wie“ des Koitus und schließen nicht selten aus dem Mitmachen der Frau, dass sie es auch wünscht und zufrieden ist [8].

\section{Methodik}

\section{Fragebogenkonstruktion}

Der Fragebogen ${ }^{1}$ sollte möglichst viele Facetten der Sexualität erfassen. Die resultierende heterogene Struktur spiegelt die unterschiedlichen Aspekte der Thematik wider und ermöglicht in den sensiblen Bereichen die Bereitstellung von Kontrollvariablen, wie beispielsweise Angaben zu negativen sexuellen Erlebnissen. Als Basisdaten und zur Einschätzung des Aktivitäts-Status-quo werden Häufigkeit und Dauer der sexuellen Handlungen, Zufriedenheit mit Häufigkeit und Dauer dieser Handlungen sowie erwünschtes Sexualverhalten in Bezug auf Häufigkeit, Dauer und Art und Weise erhoben.

Die Auswahl und Zuordnung der Items zu übergeordneten Kategorien erfolgte auf der Grundlage theoretischer Überlegungen und klinischer Beobachtungen. In den Prozess der Fragebogenentwicklung gingen Expertenratings zur Frage der adäquaten Abbildung der Zielinhalte in den einzelnen Items und den verschiedenen Bereichen ein. Die Gruppe der Experten setzte sich aus acht psychotherapeutisch erfahrenen ärztlichen und psychologischen MitarbeiterInnen der psychosomatischen Klinik sowie sechs AbsolventInnen der Ausbildung „Sexualtherapie für Paare“ zusammen. Die letztlich 173 Items

\footnotetext{
${ }^{1}$ Auf Anfrage wird der Fragebogen zugesandt.
}

wurden den folgenden neun Bereichen zugeordnet, die auch die in die Endfassung des Fragebogens aufgenommene Gliederung darstellen.

1. Häufigkeiten und Erleben sexueller Aktivitäten, 2. Orgasmushäufigkeiten und Orgasmuserleben, 3. Aktivität und Passivität in der partnerschaftlichen Sexualität, 4. sexuelle Selbstbestimmtheit, 5. Erregbarkeit und Erleben von Sexualität, 6. Kommunikation in der Partnerschaft, 7. störende Einflüsse auf sexuelle Aktivitäten, 8. körperliche Veränderungen (Schwangerschaft, Stillzeit, Menstruation) und sexuelles Erleben, 9. Gesamteinschätzung der Sexualität.

Die Skalen umfassen meist sechs Antwortmöglichkeiten. Die Mehrfachantworten enthalten alle eine zusätzliche offene Kategorie. Mit Zusatzfragen wird kontrolliert, ob die letzten drei Monate typisch oder untypisch für das Sexualleben der jeweiligen Frau sind. Weitere Kontrollfragen betreffen den Einfluss eventueller Krankheiten auf die Sexualität.

Ausgehend von den Fragen zur Häufigkeit und Dauer verschiedener sexueller Handlungen ergeben die Daten zu „wie oft?“ und „wie lange?“ quantitative Werte in Bezug auf einen festgelegten Zeitraum (in der Regel die letzten drei Monate). Aus diesen Status-quo-Daten und den gewünschten Häufigkeiten dieser sexuellen Handlungen kann eine Ist-Soll-Differenz errechnet und mit den Daten zur Zufriedenheit mit der Sexualität im Allgemeinen und mit den verschiedenen sexuellen Handlungen im Besonderen in Bezug gesetzt werden. Dabei geht es um die Frage, ob das Ausmaß der Ist-Soll-Differenz mit dem der Zufriedenheit in einem Zusammenhang steht, d.h. beispielsweise, ob hohe Quantität auf hohe Zufriedenheit schließen lässt.

Mit der Erfassung eines „Hauptziels“ sexueller Aktivitäten von Frauen soll die Orgasmusorientierung sexueller Handlungen überprüft werden. Eine Grundannahme ist, dass nicht alle Frauen orgasmusorientiert sind, d. h., dass ihre sexuelle Zufriedenheit nicht in erster Linie vom regelmäßigen Erleben des Orgasmus abhängt.

Der Vergleich der Subgruppen sexuell zufriedener und nicht zufriedener Frauen soll über die verschiedenen für die Sexualität relevanten Bereiche geführt werden. Die hieraus gewonnenen Daten sollen im Weiteren der empirischen Abstützung der theoretischen Implikationen der Fragebogenkonstruktion dienen.

\section{Stichprobe}

Die Stichprobe besteht aus technischen und Verwaltungsmitarbeiterinnen der Universitätskliniken Bonn, aus Besucherinnen von Kommunikations- und Weiterbildungszentren sowie aus Studentinnen der Psychologie und der Medizin in Bonn.

Der Fragebogen wurde im Zeitraum Mai-Juni 1997 an 250 Frauen mit primär heterosexuellem Sexualverhalten im Alter von 20-48 Jahren verteilt, die seit mindestens drei Monaten in einer Partnerschaft leben und nicht einer religiösen oder ethnischen Gruppe zugehören, welche vom mitteleuropäischen Moralkodex stark abweichende Normvorstellungen aufweist. 
Angestrebt wurde eine Stichprobengröße von $>100$. Die anonym ausgefüllten Bogen wurden von den Frauen in frankierten Rückumschlägen zurückgesendet. Die untersuchte Stichprobe umfasst 112 Probandinnen. Eine Rücklaufquote von 46\% ist als hoch zu betrachten, lag aber unterhalb unseres Erwartungsspektrums, weil die Fragebogen im Verlauf eines Gespräches nur an interessierte Frauen übergeben wurden.

Die Stichprobe umfasst die Altersspanne von 20-48 Jahren. Das Durchschnittsalter beträgt 31,4 \pm 5 ,4 Jahre. Für die statistische Auswertung wurde die Stichprobe in fünf Altersgruppen unterteilt (Tab. 1 a).

Tab. 1 a Alter

\begin{tabular}{llllll}
\hline & $\begin{array}{l}20-25 \\
\text { Jahre }\end{array}$ & $\begin{array}{l}26-30 \\
\text { Jahre }\end{array}$ & $\begin{array}{l}31-35 \\
\text { Jahre }\end{array}$ & $\begin{array}{l}36-40 \\
\text { Jahre }\end{array}$ & $\begin{array}{l}41-48 \\
\text { Jahre }\end{array}$ \\
\hline $\mathrm{n}$ & 13 & 46 & 30 & 15 & 8 \\
$\%$ & 11,6 & 41,1 & 26,8 & 13,4 & 7,1 \\
\hline
\end{tabular}

Schulbildung: 3,6\% (4) haben die Hauptschule besucht, über 90\% haben mindestens einen Realschulabschluss, 75,9\% (85) haben Abitur. Der hohe Bildungsstand ist bei der Interpretation der Daten zu berücksichtigen.

Die verschiedenen Berufe sind in Tab. 1 b zusammengefasst.

Tab. 1 b Beruf

\begin{tabular}{lcc}
\hline Beruf & $\mathrm{n}$ & $\%$ \\
\hline Angestellte & 45 & 40,2 \\
Studentinnen & 36 & 32,1 \\
Beamtinnen/Akademikerinnen & 21 & 18,9 \\
Hausfrauen & 4 & 3,6 \\
Arbeiterinnen & 3 & 2,7 \\
Selbständige & 2 & 1,8 \\
keine Angabe & 1 & 0,9 \\
\hline
\end{tabular}

Wohnsituation: 67\% (75) wohnen mit dem Partner zusammen und 33\% (37) wohnen in getrennten Wohnungen. Als ein Ergebnis der Fragebogenuntersuchung sei vorweggenommen, dass 57,1\% der Frauen sich in ihrer jetzigen Partnerschaft als absolut oder sehr glücklich ansehen; 67\% haben selten oder nie Trennungsgedanken, knapp 26\% gelegentlich.

Über die Kinderzahl gibt Tab. 1 c Auskunft. Mit durchschnittlich 0,56 Kinder pro Frau liegt die Stichprobe unter dem Bundes-

Tab.1c Kinderzahl

\begin{tabular}{llllll}
\hline & $\begin{array}{l}\text { keine } \\
\text { Kinder }\end{array}$ & $\begin{array}{l}\text { ein } \\
\text { Kind }\end{array}$ & $\begin{array}{l}\text { zwei } \\
\text { Kinder }\end{array}$ & $\begin{array}{l}\text { drei } \\
\text { Kinder }\end{array}$ & $\begin{array}{l}\text { vier } \\
\text { Kinder }\end{array}$ \\
\hline $\mathrm{n}$ & 72 & 21 & 16 & 2 & 1 \\
$\%$ & 64,3 & 18,8 & 14,3 & 1,8 & 0,9 \\
\hline
\end{tabular}

Tab.1 d Partnerschaftsdauer

\begin{tabular}{|c|c|c|c|c|c|}
\hline & $<1$ Jahr & 1-5 Jahre & 6-10 Jahre & $11-15$ Jahre & > 15 Jahre \\
\hline$n$ & 12 & 50 & 28 & 12 & 10 \\
\hline$\%$ & 10,7 & 44,6 & 25 & 10,7 & 8,9 \\
\hline
\end{tabular}

durchschnitt mit 1,2 Kindern für Frauen zwischen 15 und 49 Jahren [9].

Partnerschaftsdauer: Die Angaben zur Dauer der jetzigen Partnerschaft variieren zwischen drei Monaten und 25 Jahren. Der Mittelwert beträgt 6,3 und der Median 4,5 Jahre bei einer Standardabweichung von 5,7. Für die statistische Auswertung werden die Angaben zu fünf Gruppen zusammengefasst (Tab. 1 d).

\section{Auswertung}

Der Fragebogen wurde mit dem Statistikprogramm SPSS for Windows, Version 6.1.3 [10], ausgewertet. Das Datenmaterial wurde nach offenen und geschlossenen Fragen sortiert, ebenso nach der Art der Antwortskala. Für die Daten wurde eine Datenreduktion durch Klassenbildung oder Dichotomisierung vorgenommen. Die Rangkorrelation nach Spearman diente der Überprüfung theoretisch bedeutsamer Zusammenhänge der Variablen. Der Mann-Whitney-U-Test wurde angewendet, um zu überprüfen, in welchen Bereichen und Fragen sich die Gruppe der sexuell zufriedenen Frauen von der Gruppe der sexuell nicht zufriedenen Frauen unterscheidet.

\section{Ergebnisse der Untersuchung}

Zunächst werden in Anlehnung an die thematische Gliederung des Fragebogens die wichtigsten Ergebnisse der quantitativen Erfassung des Sexuallebens dargestellt (weitere Einzelheiten s. [11]). Eine korrelationsstatistische Analyse schließt sich an, darauf folgen die Ergebnisse des Gruppenvergleichs zwischen sexuell zufriedenen und sexuell nicht zufriedenen Frauen.

\section{Erfassung der Datenbasis zum Sexualleben}

Die Ergebnisse der quantitativen Erfassung des Sexuallebens werden überwiegend tabellarisch dargestellt. Tab. 2 greift verschiedene Aspekte sexueller Aktivitäten in der Partnerschaft auf.

Im Hinblick auf die Fähigkeit, eigene sexuelle Vorstellungen und Wünsche in der Sexualität umzusetzen, geben 45\% der Frauen an, dazu meistens oder immer in der Lage zu sein; sehr oder absolut zufrieden mit der Realisation eigener sexueller Wünsche sind 51,4\%. Als Kontrollfrage wurde die Häufigkeit erhoben, mit der Frauen in der Lage sind, im Alltag ihre eigenen Vorstellungen umzusetzen. Hier liegen die Häufigkeitsund Zufriedenheitsrate im gleichen Bereich. Die Frauen können somit in der Sexualität in gleichem Ausmaß wie in Alltagsbelangen ihre eigenen Vorstellungen umsetzen.

Die Angaben zur Masturbation (Tab. 3) lassen insgesamt darauf schließen, dass die Frauen die Selbstbefriedigung überwiegend konfliktfrei gestalten können. Dafür spricht auch, dass bei den Zufriedenheitsitems die Kategorien „kaum/gar nicht zufrieden" nicht besetzt sind. Verglichen mit den sexuellen Ak- 
Tab. 2 Sexuelle Aktivitäten mit Partner $(n=112)$.

n $\%$

\section{Zärtlichkeiten}

mit Partner täglich/mehrmals täglich zärtlich sind

Zärtlichkeitsaustausch täglich/mehrmals täglich wünschen sich

mit Zärtlichkeitshäufigkeit absolut/sehr zufrieden sind mit Art und Weise der partnerschaftlichen Zärtlichkeit absolut/sehr zufrieden sind

sexuelle Aktivitäten ohne Penetration

täglich/mehrmals wöchentlich sexuell aktiv ohne

Penetration sind

Streicheln oder Petting täglich/mehrmals wöchentlich wünschen sich

mit Häufigkeit von Streicheln/Petting absolut/sehr zufrieden sind

mit Art und Weise dieser sexuellen Aktivität absolut/ sehr zufrieden sind

Initiative

sexuelle Initiative geht deutlich häufiger vom Partner aus

gewünschte sexuelle Aktivität meistens/immer aktiv herbeiführen können

Ergreifen der Initiative fällt sehr leicht/extrem leicht

ihre Rolle bei sexuellen Handlungen als aktiv bezeichnen

Koitus

mehrmals wöchentlich als Koitushäufigkeit nennen

wöchentlich/mehrmals monatlich als Koitushäufigkeit nennen

monatlich oder seltener als Koitushäufigkeit nennen

mit Koitushäufigkeit absolut/sehr zufrieden sind

mit Koitushäufigkeit etwas/kaum/gar nicht zufrieden sind

Koitus mehrmals wöchentlich wünschen sich

Koitus wöchentlich/mehrmals monatlich wünschen sich

mit Art und Weise des Koitus absolut/sehr zufrieden sind

\section{Orgasmus}

einen Orgasmus beim Koitus erleben meistens/immer einen Orgasmus beim Koitus wünschen sich meistens/ immer

mit Orgasmushäufigkeit beim Koitus absolut/sehr zufrieden sind

mit Erleben des Orgasmus beim Koitus ziemlich zufrieden sind

mit Erleben des Orgasmus beim Koitus absolut/sehr zufrieden sind

Unterschied zwischen vaginalen und klitoralen Orgasmen empfinden

klitoralen Orgasmus bevorzugen

multiple Orgasmen bei partnerschaftlicher Sexualität erleben nie/selten

multiple Orgasmen bei partnerschaftlicher Sexualität erleben gelegentlich

Kommunikation über sexuelles Erleben/sexuelle Wünsche über Sexualität kommunizieren in Partnerschaft nie/selten/gelegentlich

mit Häufigkeit sehr zufrieden sind

über Sexualität sprechen mit Dritten nie/selten

mit Häufigkeit des Austausches absolut/sehr zufrieden sind
Tab. 3 Masturbation $(n=112)$.

\begin{tabular}{lll}
\hline & $\mathrm{n}$ & $\%$ \\
\hline nie zu masturbieren geben an & 28 & 25,0 \\
monatlich/mehrmals monatlich masturbieren & 51 & 45,6 \\
$\begin{array}{l}\text { wöchentlich bis täglich masturbieren } \\
\text { mit Masturbationshäufigkeit absolut/sehr zufrieden }\end{array}$ & 32 & 28,6 \\
$\begin{array}{l}\text { sind } \\
\text { meistens/immer einen Orgasmus bei Masturbation }\end{array}$ & 69 & 61,4 \\
erleben & & \\
$\begin{array}{l}\text { meistens/immer einen Orgasmus bei Masturbation } \\
\text { wünschen sich }\end{array}$ & 63 & 56,3 \\
$\begin{array}{l}\text { multiple Orgasmen bei Masturbation erleben selten/nie } \\
68\end{array}$ & 60,7 \\
\hline
\end{tabular}

6154,5

$65 \quad 58,5$

5549,2

tivitäten mit dem Partner lässt die Selbstbefriedigung 61,6\% der Frauen (etwas, viel oder extrem) weniger zufrieden sein. Die Diskrepanz zwischen gewünschter und realer Orgasmusrate bei der Masturbation ist geringer als bei den partnerschaftlichen sexuellen Aktivitäten.
$38 \quad 34,2$

6558,1

9383,0

$49 \quad 43,8$

$42 \quad 37,5$

$18 \quad 16,1$

$60 \quad 53,6$

$25 \quad 22,3$

$\begin{array}{ll}61 & 54,5\end{array}$

3430,4

$57 \quad 50,9$

$50 \quad 44,6$

6860,7

$55 \quad 49,1$

7264,3

$81 \quad 72,3$

$38 \quad 33,9$

$70 \quad 62,5$
$17 \quad 15,9$

3228,6

Mögliche Beeinträchtigungen des sexuellen Erlebens sind in Tab. 4 zusammengestellt. Leistungsdruck als Beeinträchtigung der Genussfähigkeit beim Geschlechtsverkehr wird seltener beklagt als Selbstbeobachtung oder Nachdenken. Durch das Leben mit Kindern unter drei Jahren ändern sich die sexuellen Bedürfnisse der Frauen sehr stark. Hier geben über $80 \%$ der Antwortenden an, dass ihre Bedürfnisse gesunken seien.

Tab. 4 Beeinträchtigung des sexuellen Erlebens $(n=112)$.

\begin{tabular}{|c|c|c|}
\hline & $\mathrm{n}$ & $\%$ \\
\hline $\begin{array}{l}\text { durch Selbstbeobachtung absolut/sehr beeinträchtigt } \\
\text { fühlen sich }\end{array}$ & 31 & 27,7 \\
\hline $\begin{array}{l}\text { sexuellen Missbrauch oder Vergewaltigung verarbeiten } \\
\text { mussten }\end{array}$ & 15 & 13,4 \\
\hline $\begin{array}{l}\text { physische oder psychische Gewalt durch einen Partner } \\
\text { erlebten }\end{array}$ & 8 & 7,1 \\
\hline $\begin{array}{l}\text { andere Erlebnisse wie beispielsweise Krankheiten } \\
\text { erleben als belastend }\end{array}$ & 10 & 8,9 \\
\hline ungewollte Schwangerschaft erlebten als belastend & 8 & 7,1 \\
\hline $\begin{array}{l}\text { keine solchen negativen Erfahrungen in Lebensge- } \\
\text { schichte hatten }\end{array}$ & 70 & 62,5 \\
\hline $\begin{array}{l}\text { während Menstruation ebenso häufig sexuellen } \\
\text { Kontakt wie sonst }\end{array}$ & 32 & 28,6 \\
\hline während Menstruation keinen sexuellen Kontakt & 18 & 16,1 \\
\hline $\begin{array}{l}\text { während Menstruation seltener sexuellen Kontakt } \\
\text { als sonst }\end{array}$ & 50 & 44,6 \\
\hline $\begin{array}{l}\text { während Menstruation häufiger sexuellen Kontakt } \\
\text { als sonst }\end{array}$ & 10 & 8,9 \\
\hline
\end{tabular}

Auf die Frage, ob es auf sie zutrifft, dass das Ziel sexueller Aktivitäten der Orgasmus ist oder ob es ein Gefühl/einen Zustand gibt, das/den zu erreichen ihnen wichtiger ist, ergeben sich die in Tab. 5 dargestellten Ergebnisse.

In der Globaleinschätzung bezeichnen sich knapp 45\% der Antwortenden als absolut oder sehr zufrieden mit ihrem Sexualle- 
Tab. 5 Erleben von Sexualität/sexuelle Selbstbestimmtheit $(n=112)$.

\begin{tabular}{|c|c|c|}
\hline & $\mathrm{n}$ & $\%$ \\
\hline Orgasmus als Ziel sexueller Aktivitäten & 49 & 43,8 \\
\hline Orgasmus spielt untergeordnete Rolle & 56 & 50,0 \\
\hline $\begin{array}{l}\text { Erleben von Geborgenheit/Nähe/Zärtlichkeit/Liebe als } \\
\text { übergeordnete Rolle bei sexuellen Aktivitäten }\end{array}$ & 41 & 36,6 \\
\hline $\begin{array}{l}\text { nach Geschlechtsverkehr meist entspannt und müde } \\
\text { fühlen sich }\end{array}$ & 91 & 81,3 \\
\hline beglückt und erfüllt nennen sich & 85 & 75,8 \\
\hline als aktiv und tatkräftig bezeichnen sich & 33 & 29,5 \\
\hline $\begin{array}{l}\text { bei seltener Nennung negativer Empfindungen fühlen } \\
\text { sich jedoch durch die erlebte Sexualität vom Partner } \\
\text { benutzt }\end{array}$ & 11 & 9,8 \\
\hline $\begin{array}{l}\text { absolut/sehr selbstbestimmt in ihrem Sexualleben } \\
\text { fühlen sich }\end{array}$ & 54 & 48,2 \\
\hline $\begin{array}{l}\text { ziemlich selbstbestimmt in ihrem Sexualleben } \\
\text { fühlen sich }\end{array}$ & 39 & 34,8 \\
\hline $\begin{array}{l}\text { mit Ausmaß an sexueller Selbstbestimmtheit absolut/ } \\
\text { sehr zufrieden }\end{array}$ & 60 & 53,6 \\
\hline $\begin{array}{l}\text { mit Ausmaß an sexueller Selbstbestimmtheit ziemlich } \\
\text { zufrieden }\end{array}$ & 27 & 24,1 \\
\hline
\end{tabular}

ben. Mit 16,8\%, die sich noch als ziemlich zufrieden bezeichnen, liegen über $61 \%$ der Antworten im oberen Bereich der Antwortskala (absolut, sehr und ziemlich zufrieden).

\section{Korrelationsstatistische Analyse}

Die Zusammenhänge einzelner Items mit dem Globalrating der sexuellen Zufriedenheit dienen der Überprüfung der gemeinsamen Varianz dieser aus Theoriebildung und klinischen Beobachtungen abgeleiteten Aspekte der sexuellen Gesamtbefindlichkeit. Die sexuelle Zufriedenheit korreliert zwar eindeutig mit den Items der sexuellen Aktivität (Koitus etc.), unerwartet hoch sind jedoch die Korrelationen mit den nicht so sehr die sexuelle Aktivität, sondern mehr die Partnerschaft charakterisierenden Variablen „Glücklichsein in der Beziehung“ und „Übereinstimmung der sexuellen Wünsche“ (Tab. 6). Die gemeinsame Varianz mit diesen Variablen beträgt 41 bzw. 33\%. Ähnlich hohe Korrelationskoeffizienten errechnen sich zur Häufigkeit des Entstehens „großer Lust auf Sexualität" in der Partnerschaft $(r=.55)$. Dieses Ergebnis wird durch die hohe negative Korrelation zu der Inzidenz des Libidoverlustes während der sexuellen Aktivität $(\mathrm{r}=-.56)$ bestätigt. Die aus den Sexualanamnesen und der Literatur bekannte hohe Bedeutung von kognitiven Störeinflüssen für sexuelle Aktivität und Zufriedenheit kann in der untersuchten, nicht klinischen Stichprobe durch die hohe negative Korrelation zwischen sexueller Zufriedenheit und Selbstbeobachtung bzw. Leistungsdruck $(r=-.47)$ ebenfalls nachgewiesen werden. Die nur geringen Korrelationen $(\mathrm{r}=.27, \mathrm{r}=.16)$ zwischen der sexuellen Zufriedenheit und der Orgasmushäufigkeit bestätigen die der Fragebogenkonstruktion zugrunde liegende Hypothese, welche die Orgasmusorientierung der Frau infrage stellt.
Tab. 6 Korrelationen mit „sexueller Zufriedenheit“; Spearman-Rangkorrelation, 2-seitige Irrtumswahrscheinlichkeit.

\begin{tabular}{|c|c|c|}
\hline Item & $r$ & $\mathrm{p}$ \\
\hline 8. Häufigkeit sexueller Aktivität ohne Penetration & .34 & .000 \\
\hline 15. Koitushäufigkeit & .50 & .000 \\
\hline 73. Häufigkeit des Empfindens „großer Lust“ & .55 & .000 \\
\hline $\begin{array}{l}\text { 29. Orgasmushäufigkeit bei sexuellen Aktivitäten } \\
\text { ohne Penetration }\end{array}$ & .27 & .004 \\
\hline 33. Orgasmushäufigkeit bei Koitus & .16 & .098 \\
\hline $\begin{array}{l}\text { 48. Fähigkeit, sexuelle Wünsche und Vorstellungen } \\
\text { umzusetzen }\end{array}$ & .45 & .000 \\
\hline $\begin{array}{l}\text { 59. Übereinstimmung mit sexuellen Wünschen des } \\
\text { Partners }\end{array}$ & .58 & .000 \\
\hline A. Glücklichsein in der Beziehung & .64 & .000 \\
\hline $\begin{array}{l}\text { 91. Häufigkeit von Selbstbeobachtung bei sexuellen } \\
\text { Aktivitäten }\end{array}$ & -.47 & .000 \\
\hline $\begin{array}{l}\text { 93. Häufigkeit von Leistungsdruck bei sexuellen } \\
\text { Aktivitäten }\end{array}$ & -.47 & .000 \\
\hline $\begin{array}{l}\text { 95. Häufigkeit von Nachdenken während sexueller } \\
\text { Aktivitäten }\end{array}$ & -.22 & .000 \\
\hline $\begin{array}{l}\text { 125. Häufigkeit schwindender/nachlassender Lust } \\
\text { bei sexuellen Aktivitäten }\end{array}$ & -.56 & .000 \\
\hline 71. sexuelle Selbstbestimmtheit & .50 & .000 \\
\hline
\end{tabular}

Gruppenvergleich: Sexuell zufriedene vs. sexuell nicht zufriedene Frauen

Anhand eines Extremgruppenvergleiches wird mit dem MannWhitney-U-Test überprüft, ob der Fragebogen zwischen sexuell zufriedenen und sexuell nicht zufriedenen Frauen diskriminieren kann. Die Gruppe der zufriedenen Frauen bilden diejenigen, die sich als absolut oder sehr zufrieden mit ihrem Sexualleben bezeichnen $(n=59)$, die Gruppe der nicht zufriedenen diejenigen, die sich mit ihrem Sexualleben als etwas, kaum oder gar nicht zufrieden bezeichnen $(n=23$, s.a. Tab. 7). Hinsichtlich des Alters, der Partnerschaftsdauer und der Kinderzahl unterscheiden sich die beiden Gruppen statistisch nicht voneinander. 75\% der Items diskriminieren zwischen diesen beiden Gruppen.

Häufigkeit sexueller Aktivitäten und Orgasmushäufigkeit: Zufriedene Frauen geben für den Koitus, die sexuellen Aktivitäten mit dem Partner ohne Penetration und für den Zärtlichkeitsaustausch signifikant höhere Häufigkeitswerte an als nicht zu-

Tab. 7 Daten für die Überprüfung der Vergleichbarkeit der Extremgruppen.

\begin{tabular}{|c|c|c|c|c|}
\hline & \multicolumn{2}{|c|}{$\begin{array}{l}\text { sexuell zufriedene } \\
\text { Frauen }(n=59)\end{array}$} & \multicolumn{2}{|c|}{$\begin{array}{l}\text { sexuell nicht zufrie- } \\
\text { dene Frauen }(n=23)\end{array}$} \\
\hline & $\mathrm{M}$ & SD & $\mathrm{M}$ & SD \\
\hline Alter (Jahre) & 30,9 & 5,8 & 30,6 & 3,2 \\
\hline $\begin{array}{l}\text { Dauer der Partnerschaft } \\
\text { (Jahre) }\end{array}$ & 6,0 & 5,5 & 6,1 & 4,3 \\
\hline Kinderzahl & 0,5 & 0,8 & 0,7 & 0,9 \\
\hline
\end{tabular}


Tab. 8 Signifikanz der Gruppenunterschiede bezüglich sexueller Aktivitäten.

\begin{tabular}{|c|c|}
\hline Item & $\begin{array}{l}\text { Signifikanz- } \\
\text { niveau }\end{array}$ \\
\hline 1. Häufigkeit des Zärtlichkeitsaustausches & $*(.0130)$ \\
\hline 2. Zufriedenheit mit Zärtlichkeitshäufigkeit & $* * *(.0001)$ \\
\hline 4. Dauer des Zärtlichkeitsaustausches & n.s. \\
\hline $\begin{array}{l}\text { 5. Zufriedenheit mit Dauer des Zärtlichkeitsaus- } \\
\text { tausches }\end{array}$ & $* * *(.0003)$ \\
\hline $\begin{array}{l}\text { 7. Zufriedenheit mit Art und Weise des Zärtlich- } \\
\text { keitsaustausches }\end{array}$ & $* * *(.0001)$ \\
\hline 8. Häufigkeit sexueller Aktivitäten ohne Penetration & $* *(.004)$ \\
\hline $\begin{array}{l}\text { 9. Zufriedenheit mit Häufigkeit sexueller Aktivitäten } \\
\text { ohne Penetration }\end{array}$ & $* * *(.0000)$ \\
\hline 11. Dauer sexueller Aktivitäten ohne Penetration & n.s. \\
\hline $\begin{array}{l}\text { 12. Zufriedenheit mit Dauer sexueller Aktivitäten } \\
\text { ohne Penetration }\end{array}$ & $* * *(.0000)$ \\
\hline $\begin{array}{l}\text { 14. Zufriedenheit mit Art und Weise sexueller } \\
\text { Aktivitäten ohne Penetration }\end{array}$ & $* * *(.0000)$ \\
\hline 15. Koitushäufigkeit & $* * *(.0000)$ \\
\hline 16. Zufriedenheit mit Koitushäufigkeit & $* * *(.0000)$ \\
\hline 18. Koitusdauer & n.s. \\
\hline 19. Zufriedenheit mit Koitusdauer & $* * *(.0000)$ \\
\hline 21. Zufriedenheit mit Art und Weise des Koitus & $* * *(.0000)$ \\
\hline 22. Masturbationshäufigkeit & n.s. \\
\hline 23. Zufriedenheit mit Masturbationshäufigkeit & n.s. \\
\hline 25. Masturbationsdauer & n.s. \\
\hline 26. Zufriedenheit mit Masturbationsdauer & n.s. \\
\hline $\begin{array}{l}\text { 28. Zufriedenheit mit Art und Weise der } \\
\text { Masturbation }\end{array}$ & $*(.0261)$ \\
\hline
\end{tabular}

friedene Frauen (Tab. 8). Das gilt ebenfalls für die Häufigkeit und die Zufriedenheit mit dem Orgasmuserleben bei partnerschaftlicher Sexualität. Für die Dauer der unterschiedlichen sexuellen Aktivitäten ergeben sich keine signifikanten Unterschiede. Auch die Daten zur Masturbation liefern bezüglich Häufigkeit und Dauer keine signifikanten Gruppenunterschiede zwischen sexuell zufriedenen Frauen und nicht zufriedenen Frauen.

Aktivität und Passivität in der Partnerschaft: Weiter beschreiben sich die zufriedenen Frauen als signifikant aktiver und initiativer in ihrer sexuellen Rolle.

Erleben von Sexualität; Störungen und Beeinträchtigungen: Sexuell zufriedene Frauen verspüren signifikant häufiger als nicht zufriedene Frauen das „Gefühl großer Lust auf Sexualität" mit ihrem Partner und sind signifikant schneller erregt. Hinsichtlich der Häufigkeit des Erlebens multipler Orgasmen unterscheiden sich die Gruppen nicht. Bezüglich negativer Erlebnisqualitäten während sexueller Kontakte mit dem Partner fallen die Vergleiche signifikant aus: Durch Leistungsdruck, Selbstbeobachtung und Nachdenken über die eigene Attraktivität und das Lustempfinden des Partners fühlen sich sexuell nicht zufriedene Frauen häufiger und stärker beeinträchtigt als zufriedene Frauen. Das Nachlassen oder Verschwinden der Lust beim Geschlechtsverkehr betrifft die Zufriedenen signifikant seltener als die nicht Zufriedenen. Hinsichtlich der Beein-
Tab.9 Signifikanz der Gruppenunterschiede bezüglich Kommunikation.

Item

Signifikanz-

niveau

102. Wissen über eigene Sexualität

$*(.0111)$

103. Zufriedenheit mit Wissen über eigene Sexualität

$* * *(.0000)$

104. Wissen über Sexualität des Partners

$*(.0148)$

105. Zufriedenheit mit Wissen über Sexualität des Partners

106. tägliche Gesprächszeit mit Partner $\quad * *(.0029)$

107. Zufriedenheit mit täglicher Gesprächszeit mit $\quad * * *(.0000)$ Partner

108. Häufigkeit von Reden mit Partner über sexuelle $\quad * * *(.0007)$ Ideen, Wünsche, Erleben

109. Zufriedenheit mit Häufigkeit von Reden über $\quad * * *(.0000)$ sexuelle Ideen/Wünsche/Erleben

110. Wichtigkeit von nonverbaler Verständigung n.s. während sexueller Aktivitäten

111. Zufriedenheit mit nonverbaler Verständigung mit ${ }^{* * *}(.0002)$ Partner während sexueller Aktivitäten

112. Häufigkeit von Reden über sexuelle Vor-

n.s. stellungen/Wünsche während sexueller Aktivitäten

113. Zufriedenheit mit Redehäufigkeit über sexuelle Vorstellungen/Wünsche während sexueller Aktivitäten

114. Häufigkeit von Reden über Empfindungen und n.s. Gefühle während sexueller Aktivitäten

115. Zufriedenheit mit Redehäufigkeit über Empfin- $\quad * * *(.0009)$ dungen und Gefühle während sexueller Aktivitäten

116. Häufigkeit von Austausch mit Dritten über Sexualität

117. Zufriedenheit mit Häufigkeit von Austausch mit Dritten über Sexualität

n.s.

** $(.0062)$

trächtigung durch negative sexuelle Erlebnisse in ihrer Lebensgeschichte unterscheiden sich die Gruppen nicht.

Kommunikation in der Partnerschaft: Verglichen mit den sexuell nicht zufriedenen Frauen geben die zufriedenen Frauen an, mehr sowohl über ihre eigene als auch über die Sexualität ihres Partners zu wissen (Tab. 9). Außerdem kommunizieren zufriedene Frauen signifikant häufiger mit ihren Partnern „außerhalb des Bettes“. Kein Unterschied ergibt sich jedoch beim Kommunikationsverhalten während sexueller Aktivitäten, wobei die Zufriedenheit mit dem Kommunikationsverhalten die Gruppen signifikant trennt.

Gesamteinschätzung der Sexualität: Erwartungsgemäß unterscheiden sich die zufriedenen von den nicht zufriedenen Frauen der Stichprobe signifikant durch eine glücklichere Beziehung und seltenere Gedanken an Trennung.

\section{Diskussion}

Die Bereitschaft, über die eigene Sexualität Auskunft zu geben, unterscheidet Menschen auch heute noch stark voneinander. Ob und in welcher Hinsicht sich TeilnehmerInnen an einer Befragung zur eigenen Sexualität von NichteilnehmerInnen unterscheiden, ist bisher nicht bekannt. Die Frauen unserer Un- 
tersuchung weisen einen hohen Bildungsstand auf; möglicherweise unterscheiden sie sich von der Normalpopulation auch durch eine größere Zufriedenheit mit ihrem Sexualleben, was die Auskunftsbereitschaft weiter erhöht haben könnte. Auf jeden Fall handelt es sich um eine Stichprobe von Frauen, die zu fast zwei Drittel keine Kinder haben, was neben dem hohen Bildungsstand bei der Interpretation der Daten zu bedenken ist.

Die Konstruktion des Fragebogens bedingt, dass sich die Aussagen auf die aktuelle Partnerschaft beziehen. Es ist aber nicht sicher ablesbar, ob die Auskunft der Frauen wesentlich durch die aktuelle gelebte Partnerschaft bestimmt wird oder mehr eine zeitstabile und persönlichkeitsspezifische Einschätzung beinhaltet.

Die überprüften korrelativen Zusammenhänge mit der Globaleinschätzung der sexuellen Zufriedenheit konnte die Auswahl der betreffenden Items überwiegend bestätigen. Anhand einer deutlich größeren Stichprobe ist in nächster Zeit die Item- und Faktorenanalyse des Gesamtfragebogens geplant.

Die im Rahmen dieser Untersuchung erhobenen Daten zum Sexualverhalten stimmen trotz der geringen Stichprobengröße gut mit den Ergebnissen vergleichbarer Untersuchungen (Eichner u. Habermehl [12], Kolb [13]) überein und geben damit Hinweise auf eine hinreichende externe Validität des Fragebogens.

Die in unserer Studie ermittelte hohe Wunschrate bezüglich des koitalen Orgasmus scheint die Orgasmusorientierung anderer Untersuchungen zu bestätigen. Andererseits nennen über die Hälfte der von uns erfassten Frauen den Orgasmus nicht als ihr Ziel oder ihre favorisierte Empfindung beim Geschlechtsverkehr. Für etwa 37\% der Frauen ist die emotionale und körperliche Nähe zu ihrem Partner wichtiger als das Erleben des Orgasmus. Dies bedeutet, dass der Orgasmus beim Geschlechtsverkehr zwar meist gewünscht, aber nicht als übergeordnetes Ziel betrachtet wird.

Bezüglich der Kommunikation in der Partnerschaft wurden die impliziten Annahmen des Fragebogens bestätigt. Zufriedene Frauen kommunizieren mehr mit ihren Partnern als nicht zufriedene Frauen. Für die Verständigung während sexueller Aktivitäten ergeben sich keine Unterschiede. Während sexueller Aktivitäten verläuft die Verständigung häufiger nonverbal; Probleme oder auch Wünsche werden offenbar nicht versprachlicht, möglicherweise um zu verhindern, dass mit der sprachlichen Formulierung das Erleben auf die kognitive Ebene verschoben wird.

Deutliche negative Auswirkungen auf die sexuelle Zufriedenheit haben Schwangerschaft, Stillzeit und das Leben mit Kleinkindern. Dieser Befund bestätigt die Ergebnisse anderer Autorenzitate; die meisten empirischen Untersuchungen zu den Einflüssen der Elternschaft auf die Paarbeziehung zeigen eine Verschlechterung wesentlicher Paar-Beziehungsaspekte durch die Geburt eines Kindes an (Engfer et al. [14], Meyer [15]). In Sexualanamnesen sollten daher Schwangerschaft, Stillzeit und die Versorgung von kleinen Kindern sowie eventuelle Bewältigungsstrategien von Frauen und Paaren stärkere Beachtung finden.
Sexuelle Aktivitäten während der Menstruation werden von $73,1 \%$ der Frauen als unangenehmer als sonst erlebt. Dennoch haben 37\% der Frauen während der Menstruation ebenso häufig oder sogar häufiger als sonst sexuelle Kontakte. Hier ergeben sich möglicherweise Überschneidungen mit den Frauen, die nur schwer die Wünsche des Partners ablehnen können.

Über $12 \%$ der Frauen der vorliegenden Stichprobe mussten einen sexuellen Missbrauch oder eine Vergewaltigung verarbeiten. Der Befund, dass sich die sexuell nicht zufriedenen Frauen von den sexuell zufriedenen Frauen nicht hinsichtlich der Beeinträchtigung durch negative sexuelle Erlebnisse in der Lebensgeschichte unterscheiden, überrascht daher zunächst. Sexuelle Störungen zählen neben Ängsten und Depressionen nach Beitchman et al. [16] zu den möglichen langfristigen Auswirkungen eines sexuellen Missbrauchs. Studien zu protektiven Entwicklungsfaktoren in Kindheit und Jugend zeigen jedoch, dass biografische Belastungen durch spätere positive Erfahrungen vermutlich ausgeglichen bzw. korrigiert werden können. Zu den Schutzfaktoren im Hinblick auf die Entstehung psychischer und psychosomatischer Krankheiten sind nach Egle et al. [17] neben einem robusten, aktiven und kontaktfreudigen Temperament u.a. eine verlässlich unterstützende Bezugsperson im Erwachsenenalter und eine überdurchschnittliche Intelligenz zu rechnen (Bender u. Lösel [18]). Der vergleichsweise hohe Bildungsstand unserer Stichprobe lässt eine überdurchschnittliche Intelligenz annehmen. Die befragten Frauen leben alle in einer Partnerschaft, über die Hälfte von ihnen sieht sich in ihrer jetzigen Partnerschaft als absolut oder sehr glücklich an und wohnt mit dem Partner zusammen, was auf eine verlässlich unterstützende Bezugsperson schließen lässt. Auch andere Sozialdaten wie die mehrjährige mittlere Partnerschaftsdauer sprechen dafür, dass ein eventuelles negatives Erlebnis in der Lebensgeschichte potenziell durch spätere positive Erfahrungen ausgeglichen oder gemildert werden konnte.

Aus dem Extremgruppenvergleich lässt sich folgende sexualtherapeutische Konsequenz ableiten. Sexuelle Zufriedenheit korreliert mit der in der Partnerschaft realisierten Selbstbestimmtheit, der Befriedigung der Kommunikationswünsche innerhalb der Partnerschaft und des Zärtlichkeitsbedürfnisses. Für die Sexualtherapie bedeutet dies, dass die Fokussierung auf konkretes Sexualverhalten mit einer gleichrangigen Beachtung dieser Voraussetzungen für das Erleben individueller sexueller Zufriedenheit zu verbinden ist.

\section{Literatur}

${ }^{1}$ Davis CM, Yarber WL, Bauserman R, Schreer G, Davis SL (eds). Handbook of Sexuality-Related Measures. Thousand Oaks, CA: Sage, 1998

${ }^{2}$ Schröder B, Hahlweg K, Hank G, Klann N. Sexuelle Unzufriedenheit und Qualität der Partnerschaft (befriedigende Sexualität gleich gute Partnerschaft?). Z Klin Psychol 1994; 23: 178-187

${ }^{3}$ Strauß B, Heim D. Standardisierte Verfahren in der empirischen Sexualforschung. Eine tabellarische Übersicht. Z Sexualforsch 1999; 12: 187-236

${ }^{4}$ Levine SB. Sexual Life: A Clinicians's Guide. New York: Plenum Press, 1992

${ }^{5}$ Hite S. Weibliche Sexualität. München: Goldmann, 1978

${ }^{6}$ Hite S. Der neue Hite-Report. Frauen und Liebe. München: Bertelsmann, 1987 
${ }^{7}$ von Sydow K. Psychosexuelle Entwicklung im Lebenslauf: Eine biographische Studie bei Frauen der Geburtsjahrgänge 1895 bis 1936. Regensburg: Roderer, 1991

${ }^{8}$ Schnabl S. Des einen Lust, des anderen Last. Sexualmedizin 1995; 3: $82-86$

${ }^{9}$ Statistisches Bundesamt. Statistisches Jahrbuch 1991 für das vereinte Deutschland. Wiesbaden: Statistisches Bundesamt, 1995

10 Brosius G, Brosius F. SPSS Basis System and Professional Statistics. Bonn: Thomas, 1995

${ }^{11}$ Hoppe $\mathrm{C}$. Theoretische und praktische Erstellung eines Fragebogens zur sexuellen Zufriedenheit von Frauen. Universität Bonn: Psychologische Diplomarbeit, 1997

12 Eichner K, Habermehl W. Der Ralf-Report. Hamburg: Hoffman \& Campe, 1978

${ }^{13}$ Kolb I. Das Kreuz mit der Liebe - Der Mythos der sexuellen Befreiung. Hamburg: Gruner \& Jahr, 1981

${ }^{14}$ Engfer A, Gavranidou M, Heinig L. Veränderungen in Ehe und Partnerschaft nach der Geburt von Kindern; Ergebnisse einer Längsschnittstudie. Verhaltensmodifikation Verhaltensmed 1998; 4: $297-311$

${ }^{15}$ Meyer HJ. Partnerschaft und emotionale Befindlichkeit von Eltern nach der Geburt ihres ersten und zweiten Kindes. In: Cierpka M, Nordmann E (Hrsg): Wie normal ist die Normalfamilie? Berlin: Springer, 1998: 43-62

${ }^{16}$ Beitchman JH, Zucker KJ, Hood JE, Da Costa GA, Akman D, Cassavia $\mathrm{E}$. A review of the long-term effects of child sexual abuse. Child Abuse Neglect 1992; 16: $101-118$

${ }^{17}$ Egle UT, Hoffmann SO, Steffens M. Pathogene und protektive Entwicklungsfaktoren in Kindheit und Jugend. In: Egle UT, Hoffmann SO, Joraschky P (Hrsg): Sexueller Missbrauch, Misshandlung, Vernachlässigung. Stuttgart: Schattauer, 1997: 3-20

18 Bender D, Lösel F. Risiko- und Schutzfaktoren in der Genese und der Bewältigung von Misshandlung und Vernachlässigung. In: Egle UT, Hoffmann SO, Joraschky P (Hrsg): Sexueller Missbrauch, Misshandlung, Vernachlässigung. Stuttgart: Schattauer, 1997: $35-53$
Dipl.-Psych. Sabine Büsing

Klinik und Poliklinik für Psychosomatische Medizin und Psychotherapie der Universität Bonn Sigmund-Freud-Straße 25 53105 Bonn 